\title{
Mumps caused by paraquat-induced poisoning: A case report
}

\author{
XIAOYAN XU, ZHONGKAI YU, YONGGUANG LIANG, HENGQIANG GAO, YILIN LIU and YUNBEI YU
}

Department of Pharmacy, Liaocheng People's Hospital, Liaocheng, Shandong 252000, P.R. China

Received May 7, 2015; Accepted May 27, 2016

DOI: $10.3892 /$ etm.2016.3982

\begin{abstract}
Paraquat (PQ) is a highly toxic herbicide that harms the liver, kidney, lungs and heart, and results in a variety of complications. The majority of patients with severe PQ poisoning may succumb to multiple organ failure, and the mortality rate is high. Although a large number of studies have been performed investigating PQ poisoning, cases of mumps caused by $\mathrm{PQ}$-induced poisoning are rare. In the present case report, a 45-year-old female who ingested PQ was admitted to the Emergency Department of Liaocheng People's Hospital (Liaocheng, China). During the development of the disease, mumps was caused by a cavity ulcer following PQ poisoning. To the best of our knowledge, cases of mumps following PQ poisoning are rare.
\end{abstract}

\section{Introduction}

Paraquat (PQ) is widely used in agricultural production as a herbicide, and is very toxic to humans and animals (1). Committing suicide is the primary reason why PQ is ingested in humans worldwide, and this trend is increasing (2). PQ combines with human tissue cells rapidly once it enters the human body and induces strong oxidization, causes lipid peroxidation and generates a large quantity of oxygen free radicals, which can damage cells and result in apoptosis and necrosis (3). PQ has the most serious toxic effects on the lung, and can cause pulmonary congestion, hemorrhage, edema and fibrosis; as a result, patients typically succumb to suffocation (4). Furthermore, PQ has a poisonous effect on the liver, kidney and pancreas (5).

The China Toxicology Medical Association has regarded the PQ poisoning as a hotspot, as the high mortality rate and the complex complications. In addition to the traditional effects on the liver, gastrointestinal tract, lung and kidney, there are also something damage on skin lesions and the pancreas $(6,7)$.

PQ poisoning is difficult to treat clinically due to the lack of effective treatments; these have not been developed, as its

Correspondence to: Dr Yongguang Liang, Department of Pharmacy, Liaocheng People's Hospital, 67 Dongchang West Road, Liaocheng, Shandong 252000, P.R. China

E-mail: liangyg1001@163.com

Key words: paraquat, poisoning, mumps underlying mechanisms are not fully understood (3). The lethal dose of PQ is a few milliliters; one mouthful is approximately $30 \mathrm{ml}$, which is markedly larger than the lethal dose $(8,9)$. As the increasing mortality of PQP (10), there exists a comprehensive treatment method, which is the gastric lavage, catharsis, fluid infusion, blood purification and the protection of vital organs (lung, liver, kidney, heart, gastrointestinal (11).

\section{Case report}

A 45-year-old female farmer was admitted to Liaocheng People's Hospital (Liaocheng, China) at 22:00 on August 17, 2014, after she ingested $50 \mathrm{ml} \mathrm{PQ} 4 \mathrm{~h}$ previously. The following results were obtained by physical examination: Temperature, $36.3^{\circ} \mathrm{C}$; pulse, 92 beats $/ \mathrm{min}$; respiratory rate, 16 breaths/min; blood pressure, $118 / 83 \mathrm{mmHg}$; conscious with malaise; no ulcers or erosion in the oral cavity or on the tongue body mucosa; and the heart, lungs and abdomen showed no obvious abnormalities. Laboratory examinations, including blood analysis, liver and renal function analysis, myocardial enzyme, serum amylase (AMS) and blood sugar analysis, were not abnormal. Treatments administered to the patient included gastrointestinal purification and mannitol for catharsis, methylprednisolone pulse therapy (500-1,000 mg for 3-5 days and according to the security of the disease gradually reduced), protection of organ function (fructose phosphate to protect the heart, magnesium isoglycyrrhizinate to protect the liver, Chinese medicine Jinshuibao capsule to protect kidney and proton pump inhibitor to protect the gastrointestinal tract) and hemoperfusion (11).

The patient began to develop pain in the pharynx and tongue on the second day of admission (August 18th, 2014). This pain worsened on August 20th, 2014, and oral cavity examination revealed a large quantity of ulcers in the bilateral lip, cheek and tongue mucosa, and the overlying necrotic tissue of the mucosa. On August 23rd, the patient felt that the pain in the pharynx and tongue body had improved; however, upon eating breakfast, the right side of the patient's face became oncotic suddenly and rapidly. Physical examination showed the following: Temperature, $36.6^{\circ} \mathrm{C}$; the right parotid gland was inflamed; mild tenderness of the right parotid gland; the left parotid and submandibular gland was not inflamed; the lips and oral cheek mucous membrane had a large number of ulcers; there was no liquid outflow from the parotid gland orifice, which was not inflamed (Fig. 1); and no abdominal pain was present. AMS expression levels were $490 \mathrm{IU} / 1$, which increased markedly from the original value of $56 \mathrm{IU} / 1$. 


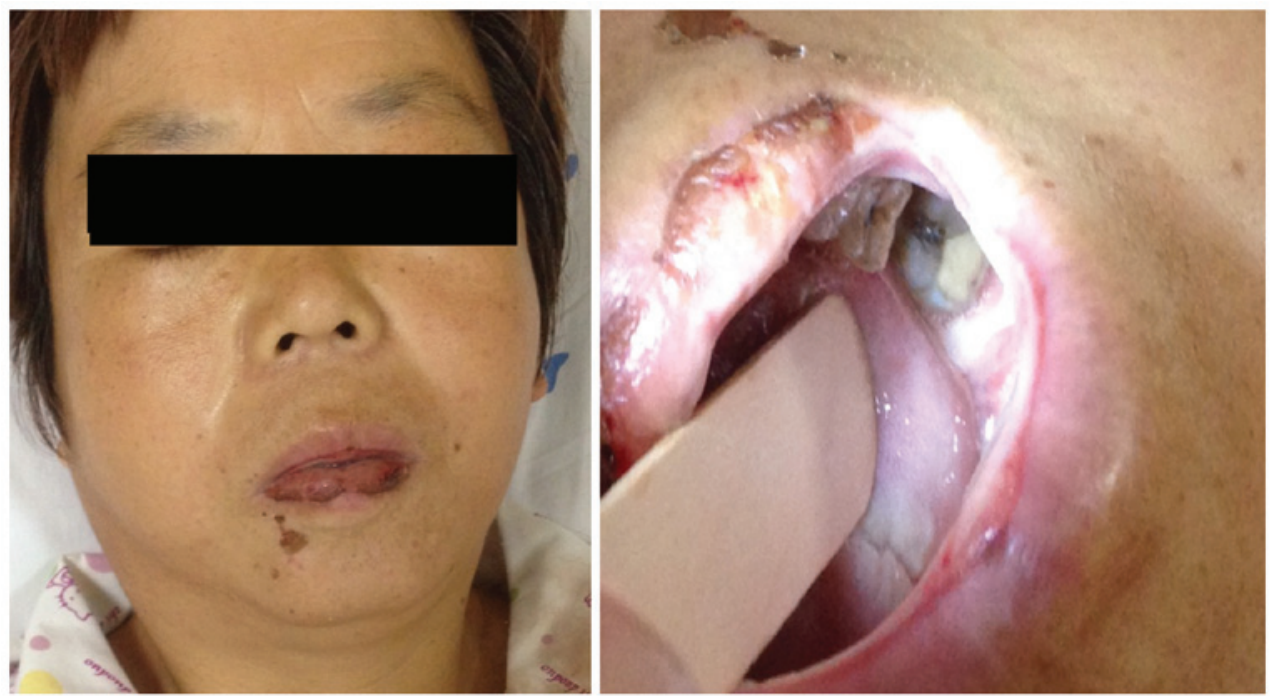

Figure 1. On August 23rd, 2014 (the first day of developing mumps), the right parotid gland was intumescent with some tenderness, and the lip and cheek mucous membrane had a large number of ulcers.

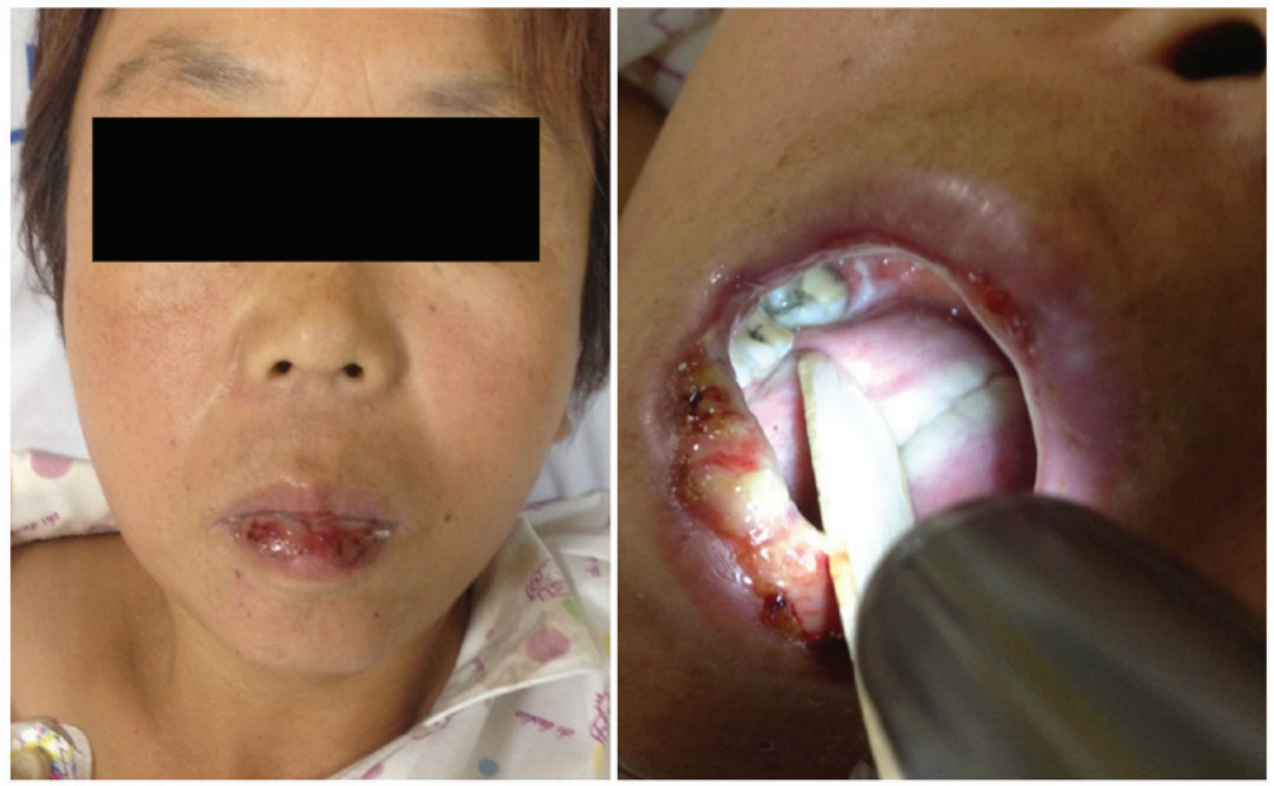

Figure 2. On August 24th, 2014 (the second day of developing mumps), the right parotid gland swelling was reduced, and tenderness was not evident. However, the lip and oral cheek mucous membrane ulcers still remained.

Leukocyte, neutrophil and lymphocyte counts were normal; therefore, it was concluded that mumps was induced following the poor flow of parotid gland secretory fluid, which was caused by the blockage of the right parotid gland duct caused by a cavity ulcer following PQ poisoning.

Mumps were treated using gentamicin solution mouthwash (Shandong Mainland Pharmaceutical Co., Ltd., Shandong, China), montmorillonite powder (Yangtze River Pharmaceutical Group Co., Ltd., Jiangsu, China) for topical application on cavity ulcers and infrared irradiation of the parotid gland. On August 24th, 2014, the right parotid gland swelling was relieved, and tenderness was not evident (Fig. 2). The AMS was $152 \mathrm{IU} / 1$ and the urine amylase (UAMY) was 100 IU/1; leukocyte, neutrophil and lymphocyte counts remained normal, at $6.79,3.76$ and $2.37 \times 10^{9}$ cells $/ 1$, respectively. On
August 25, 2014, the right parotid gland swelling had disappeared completely, and no tenderness was present; the bilateral lip and oral buccal mucosa ulcers were evidently improved. The AMS was $72.1 \mathrm{IU} / 1$ and the UAMY was $67 \mathrm{IU} / 1$, both of which had returned to normal. At this point, infrared treatment was discontinued; however, gentamicin solution mouthwash and montmorillonite powder were continued to be administered in order to treat the oral ulcers until they had healed completely. The patient underwent a total of 17 days of hospitalization, and was discharged on September 4, 2014. To date, the patient is recovered well and does not feel any discomfort. The results of AMS, UAMY and blood analysis are presented in Table I. The primary indexes of liver and renal function are presented in Table II. The health of the patient has remained unchanged since the submission of this case report for publication. 
Table I. Results of AMS, UAMY and blood analysis in August, 2014.

\begin{tabular}{lccc}
\hline Testing index (reference ranges) & August 23rd & August 24th & August 25th \\
\hline AMS (0-130 IU/l) & 490 & 152 & 72.1 \\
UAMY (0-330 IU/1) & Untested & 100 & 67 \\
Leucocyte count $\left(4-10 \times 10^{9} / 1\right)$ & 5.90 & 6.79 & 8.09 \\
Neutrophilic granulocyte count $\left(2-7 \times 10^{9} / 1\right)$ & 3.79 & 3.76 & 4.66 \\
Lymphocyte count $\left(0.8-4.0 \times 10^{9} / 1\right)$ & 1.55 & 2.37 & 2.78 \\
\hline
\end{tabular}

AMS, serum amylase; UAMY, urine amylase.

Table II. Primary indexes of liver and renal function in August, 2014.

\begin{tabular}{lccc}
\hline Testing index (reference ranges) & August 18th & August 23rd & August 27th \\
\hline ALT (0-40 IU/l) & 45 & 40 & 21 \\
AST (0-40 IU/l) & 41 & 37 & 15 \\
ALP (40-150 IU/l) & 48 & 28 & 30 \\
BUN $(1.6-8.21 \mathrm{mmol} / \mathrm{l})$ & 3.4 & 4.5 & 1.8 \\
$\operatorname{SCr}(53-97 \mu \mathrm{mol} / \mathrm{l})$ & 41 & 55 & 50 \\
\hline
\end{tabular}

ALT, alanine transaminase; AST, aspartate aminotransferase; alkaline phosphatase; BUN, blood urea nitrogen; SCr, serum creatinine.

\section{Discussion}

Mumps includes epidemic parotitis and pyogenic parotitis; epidemic parotitis, which is known as viral mumps, is caused by the mumps virus (12). Children and adolescents are particularly susceptible to developing mumps, as they have the mumps virus infectious contact history (13). Pyogenic parotitis is caused by bacterial infection (14), and the clinical manifestation includes that one side parotid gland of patient is inflamed, the patient often feels fever, and the parotid gland appears red, swollen, hot and painful. Pus exits the parotid gland duct when pressure is applied to the parotid gland. Laboratory examinations have demonstrated that leucocyte and neutrophil counts, and AMS and urine amylase, increase significantly during mumps infection $(15,16)$.

Cases of mumps caused by pesticide-induced poisoning are rare. In the present study, the development of mumps following PQ poisoning may be related to the following factors: i) PQ has a strong corrosive effect on human skin and mucosa (17), therefore, the majority of patients with PQ poisoning will develop oral cavity, pharyngeal and tongue mucosa erosion and ulcers, which are difficult to heal. This is an important factor in the development of mumps. ii) In the disease process of the patient in the current study, the buccal ulcers caused swelling of the cell wall near the parotid duct, inflammatory cell infiltration around the parotid duct and interstitial edema. This will eventually result in the blockage of the parotid duct, which will cause a reduction in parotid secretion outflow. In addition, blockage of the parotid duct may cause amylase retention, which restricts amylase excretion from the parotid duct, resulting in the entering of amylase into the urine and blood circulation via the lymphatic vessels (18). iii) When the patient was eating breakfast, the action of mastication may have stimulated the parotid gland to excrete a large quantity of parotid fluid by conditioned reflex. As the opening of the parotid gland had been blocked, parotid fluid would increase in the parotid gland, resulting in rapid swelling.

The healing time of the oral ulcer caused by PQ-induced poisoning is very long, which can result in the multiplication of pathogenic bacteria in the oral cavity. If gentamicin or other antibiotics are administered to treat bacteria in the mouth, the pathogenic bacteria in the oral cavity will be targeted, and pathogenic bacteria will be prevented from developing severe pyogenic parotitis. Using montmorillonite powder for the topical application of cavity ulcers can protect the wounds and promote wound healing. Physical therapy methods, such as infrared irradiation of the swollen parotid gland, help to reduce inflammation and reduce pain (19).

In conclusion, the patient in the present study developed a blocked parotid duct, but did not develop a fever or experience tenderness of the swollen parotid gland. Blood analysis, including analysis of white blood cells, neutrophils and lymphocytes, was normal throughout the patient's disease process. In addition, AMS returned to normal soon after treatment, and the patient's health improved within a short period of time. These results suggest that mumps did not develop to form pyogenic parotitis, and that treatment was timely and effective. According to the current study, a novel perspective on the treatment of PQ poisoning is presented.

\section{References}

1. Wilks MF, Fernando R, Ariyananda PL, Eddleston M, Berry DJ, Tomenson JA, Buckley NA, Jayamanne S, Gunnell D and Dawson A: Improvement in survival after paraquat ingestion following introduction of a new formulation in Sri Lanka. PLoS Med 5: e 49, 2008. 
2. Kastanaki AE, Kraniotis CF, Kranioti EF, Nathena D, Theodorakis PN and Michalodimitrakis M: Suicide by pesticide poisoning: Findings from the island of Crete, Greece. Crisis 31: 328-334, 2010.

3. Morán JM, Ortiz-Ortiz MA, Ruiz-Mesa LM and Fuentes JM: Nitric oxide in paraquat-mediated toxicity: A review. J Biochem Mol Toxicol 24: 402-409, 2010.

4. Gawarammana IB and Buckley NA: Medical management of paraquat ingestion. Br J Clin Pharmacol 72: 745-757, 2011.

5. Han J, Zhang Z, Yang S, Wang J, Yang X and Tan D: Betanin attenuates paraquat-induced liver toxicity through a mitochondrial pathway. Food Chem Toxicol 70: 100-106, 2014.

6. Xu W, Wang L, Wang Q, Li XH, Hu D, Li C, Wu T, Mohan C, Peng A and Shi Y: Paraquat Poisoning Followed by Toxic Epidermal Necrolysis: A Report of Two Cases and Published Work Review. Dermatology 231: 209-212, 2015.

7. Li Y, Wang M, Gao Y, Yang W, Xu Q, Eddleston M, Li L and $\mathrm{Yu} \mathrm{X}$ : Abnormal pancreatic enzymes and their prognostic role after acute paraquat poisoning.Sci Rep 5: 17299, 2015.

8. Bertram A, Haenel SS, Hadem J, Hoeper MM, Gottlieb J, Warnecke G, Kaschinski S, Hafer C, Kühn-Velten WN, Günther D and Kielstein JT: Tissue concentration of paraquat on day 32 after intoxication and failed bridge to transplantation by extracorporeal membrane oxygenation therapy. BMC Pharmacol Toxicol 14: 45, 2013

9. Song C, Kan B, Yu G, Jian X, Wang J and Sun J: Acute paraquat poisoning with sinus bradycardia: A case report. Exp Ther Med 8: 1459-1462, 2014.
10. Gil HW, Kang MS, Yang JO, Lee EY and Hong SY: Association between plasma paraquat level and outcome of paraquat poisoning in 375 paraquat poisoning patients. Clin Toxicol (Phila) 46: 515-518, 2008.

11. Song C, Kan B, Yu G, Jian X, Wang J and Sun J: Acute paraquat poisoning with sinus bradycardia: A case report. Exp Ther Med 8: 1459-1462, 2014

12. Cui A, Zhu Z, Chen M, Zheng H, Liu L, Wang Y, Ma Y, Wang C, Fang X, Li P, et al: Epidemiologic and genetic characteristics of mumps viruses isolated in China from 1995 to 2010. Infect Genet Evol 21: 384-390, 2014.

13. Schwab J and Baroody F: Neonatal suppurative parotitis: A case report. Clin Pediatr (Phila) 42: 565-566, 2003.

14. Kim JS, Oh JS, Kwon SH, Kim MS and Yoon YJ: Mumps Presenting as a Parotid Abscess. J Craniofac Surg 27: 233-235, 2016.

15. Chiu CH and Lin TY: Clinical and microbiological analysis of six children with acute suppurative parotitis. Acta Paediatr 85: 106-108, 1996

16. Wang Q, Wang H, Yang X, Wang K, Liu R, Li Q and Ou J: A sensitive one-step method for quantitative detection of $\alpha$-amylase in serum and urine using a personal glucose meter. Analyst 140: 1161-1165, 2015.

17. Yen TH, Lin JL, Lin-Tan DT, Hsu CW, Weng CH and Chen $\mathrm{YH}$ : Spectrum of corrosive esophageal injury after intentional paraquat ingestion. Am J Emerg Med 28: 728-733, 2010.

18. Peng WW, Li LJ and Qiao GY: Lemology. 6th edition. People's Medical Publishing House, Beijing, p82, 2006.

19. Zhang JH and Zhao N: Infrared ray in the treatment of epidemic mumps orchitis. Yi Xue Li Lun Yu Shi Jian 20: 116, 2007 (In Chinese). 